\title{
Evaluation of Some Phenolic Acids in Diabetic Neuropathy
}

\author{
Shubhangi Pawar ${ }^{1, \star}$, Aman Upaganlawar ${ }^{2}$, Chandrashekhar Upasani \\ 'Department of Pharmacology, MGV's Pharmacy College, Panchavati, Nashik, Maharashtra, INDIA. \\ 2Department of Pharmacology, SNJB's SSDJ College of Pharmacy, Chandwad, Nashik, Maharashtra, INDIA.
}

\begin{abstract}
Background: Streptozotocin (STZ) induced neuropathy is widely used preclinical model for diabetic neuropathy (DN). DN is majorly resulted due to nitrosative and oxidative stress induced by hyperglycemia. Phenolic acids are polyphenols with free radical scavenging anti-inflammatory and neuroprotectiveaction. Methods: In this study STZ $(55 \mathrm{mg} / \mathrm{kg}$, i.p) was administered in male Wistar rats and animals with hyperglycemia (fasting blood glucose $\geq 200 \mathrm{mg} / \mathrm{dl}$ ) were used for further study. Behavioural changes cold allodynia, mechanical hyperalgesia, heat hyperalgesia, mechanical allodynia were assessed weekly. Motor Nerve Conduction Velocity (MNCV) was also evaluated. Reduced Glutathione and Malondialdehyde were estimated to indicate oxidative stress. C-Reactive Protein (CRP), Insulin assay, serum electrolytes $\left(\mathrm{Na}^{+}, \mathrm{K}^{+}\right), \mathrm{TNF}-\alpha, \mathrm{IL}-6$ and INF- $\gamma$ were also estimated. Isolated sciatic nerve was histopathologically studied to support the results. Results: Treatment with syringic acid (SY) $12.5,25,50 \mathrm{mg} / \mathrm{kg}$ and of Sinapic acid (SP) 5, 10, 20 $\mathrm{mg} / \mathrm{kg}$ orally for 5 weeks has shown to reduce blood glucose level. Behavioural changes were found to be improved weekly by SY and SP in dose dependent manner. 5 weeks treatment with SY and SP was able to increase antioxidant GSH and reduce MDA level in cell. Gabapentin, SY and SP treated animals have shown decrease in TNF- $\alpha$, IL- 6 and INF- $\gamma$ and CRP. Insulin and serum electrolytes were found to be normalised in treated groups. Histopathological study has revealed protective effect of gabapentin, SY and SP by showing reverted neuronal damage. Conclusion: In conclusion, syringic acid and sinapic acid have antihyperglycemic, antioxidant and neuroprotective effect in diabetic neuropathy.
\end{abstract}

Key words: Phenolic acids, Neuropathy, Hyperalgesia, Allodynia, Nerve conduction velocity, Antioxidants, Cytokines.

\section{INTRODUCTION}

Streptozotocin (STZ) is well established and reliable to induce diabetic neuropathy. STZ is nitrosoureas antibiotic used as anticancer. STZ selectively destructs pancreatic $B$ cells at dose of 45 to $70 \mathrm{mg} / \mathrm{kg}$ (i.v or i.p) and after 3-4 days, in rats causes hyperglycemia to induce diabetes. ${ }^{1}$ Diabetic neuropathy is majorly resulted due to nitrosative and oxidative stress induced by hyperglycemia. Thus formed reactive oxygen species (ROS) can cause sensory and motor nerve conduction defects. ${ }^{2}$ In animal models of diabetes mellitus (DM), STZ is suitably used to study disease pathogenesis and its complications. ${ }^{3}$
As modern medicines prominently show adverse effects, natural drugs are safer therapeutic alternative to treat neuropathy. Various plants and their phytoconstituents are selectively studied in the treatment of neuropathy in rats. ${ }^{4}$ Phenolic acids are polyphenols, having anti-inflammatory and free radical scavenging action, have been proven as neuroprotective. ${ }^{5}$ In accordance with these effects of various phenolic acids, unravelled members of this class can be evaluated through rational research plan. Syringic acid (SY) is useful in treatment of diabetes, cardiovascular diseases, cancer and cerebral ischemia. It is having antioxidant,
Submission Date: 30-06-2020; Revision Date: 24-12-2020; Accepted Date: 03-02-2021

DOI: 10.5530/ijper.55.1s.48 Correspondence: Mrs. Shubhangi H Pawar Department of Pharmacology, MGV's Pharmacy College, Panchavati, Nashik, Maharashtra, INDIA. Phone no: +919823192936 Email id: shpawar2009@ gmail.com

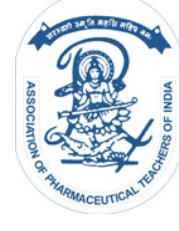

www.ijper.org 
anti-microbial, anti-inflammatory, neuroprotective and hepatoprotective activities. It effectively scavenges free radicals and reduces oxidative stress markers. ${ }^{6}$ Sinapic acid (SP) is widely used in pharmaceutical and cosmetic industries because of its potent antioxidant, anti-inflammatory, preservative and antimicrobial activity. ${ }^{7} \mathrm{SP}$ is effectively proven to prevent memory loss, counterbalancing oxidative stress and beneficial in the treatment of Alzheimer's disease. ${ }^{8}$ So the present study was undertaken to evaluate effect of SY and SP in diabetic neuropathy.

\section{MATERIALS AND METHODS}

Syringic acid and sinapic acid purchased from sigma Aldrich, USA. Ketamine (Ketamax 50) from Troikaa Pharmaceuticals, India, Xylazine (Xylaxin) from Indian Immunologicals Ltd. India and Oxytetracycline ((Terramycin) Pfizer, India. Standard drug gabapentin was supplied by Sun Pharma, India.

Research proposal was prepared as per guidelines of CPCSEA and granted approval by Institutional Animal Ethical Committee (IAEC) of SNJB's SSDJ College of Pharmacy, Chandwad, India (CPCSEA approval letter No. SSDJ/IAEC/2018/01).

Acute oral toxicity study was conducted as per OECD guidelines 425, Up and Down procedure. ${ }^{9}$ No any death observed after administration of oral dose of $2000 \mathrm{mg} / \mathrm{kg} 5$ female rats. So $\mathrm{LD}_{50}$ is concluded more than $2000 \mathrm{mg} / \mathrm{kg}$. From the literature survey, minimum therapeutic doses of Syringic acid were finalised as 12.5 , $25,50 \mathrm{mg} / \mathrm{kg} /$ day and of Sinapic acid as 5, 10, $20 \mathrm{mg} /$ $\mathrm{kg} /$ day orally. Standard drug gabapentin $300 \mathrm{mg} / \mathrm{kg} /$ day p.o. was used to compare the results.

Animals used were Wistar rats of either sex and divided into 9 groups $(n=6)$ and treated for 5 weeks as followings:

1. Negative Control: received vehicle only.

2. Positive Control: STZ $(55 \mathrm{mg} / \mathrm{kg}$, i.p.)

3. SY1: STZ ( $55 \mathrm{mg} / \mathrm{kg}$, i.p.) + Syringic acid $12.5 \mathrm{mg} / \mathrm{kg} /$ day

4. SY2: STZ (55mg/kg, i.p.) + Syringic acid $25 \mathrm{mg} / \mathrm{kg} /$ day

5. SY3: STZ (55mg/kg, i.p.) + Syringic acid $50 \mathrm{mg} / \mathrm{kg} /$ day

6. SP1: STZ ( $55 \mathrm{mg} / \mathrm{kg}$, i.p.) + Sinapic acid $5 \mathrm{mg} / \mathrm{kg} /$ day

7. SP2: STZ (55mg/kg, i.p.) + Sinapic acid $10 \mathrm{mg} / \mathrm{kg} /$ day

8. SP3: STZ (55mg/kg, i.p.) + Sinapic acid $20 \mathrm{mg} / \mathrm{kg} /$ day

9. Std: STZ (55mg/kg, i.p.) + Gabapentin $300 \mathrm{mg} / \mathrm{kg} /$ day p.o.
STZ dose in the range of 45 to $70 \mathrm{mg} / \mathrm{kg}$ develop type I DM in mammals but lower doses of STZ induce DM which may not stable and recovery from hyperglycaemia may occur. Additionally, if higher dosages are used, the death rate is found to be increased significantly. Extended research on pathological changes of DM, investigators specifically require a stable experimental model of type I DM. ${ }^{10}$ This tends to approach of dose standardization in experiment model, to choose appropriate dose of STZ which ensures minimum incidences of death and high incidences of DM. Thus in this project, from observations of pilot study, selected dose of STZ is $55 \mathrm{mg} / \mathrm{kg}$, i.p.

Induction of diabetes: STZ $(55 \mathrm{mg} / \mathrm{kg}$, i.p.) dissolved in freshly prepared $0.001 \mathrm{M}$ Citrate buffer, $\mathrm{PH} 4 . .^{11}$ After $72 \mathrm{hr}$, animals with remarkable hyperglycemia (fasting blood glucose $\geq 200 \mathrm{mg} / \mathrm{dl}$ ) ${ }^{12}$ were selected and used for further study.

Blood glucose levels: Blood glucose was monitored by using glucometer (Accu-check) to confirm hyperglycemia. Blood glucose was measured at $72 \mathrm{hr}$ and weekly thereafter till the end of treatment.

Behavioural study: In diabetic neuropathy behavioural biomarkers such as dysesthesia, hyperalgesia, allodynia and with motor in co-ordination are used to characterize peripheral nerve injury. ${ }^{13}$

a) Mechanical Allodynia (Von Frey test): Individually, rats were placed on elevated maze in acrylic cage and adopted for test environment for at least $15 \mathrm{~min}$. From below the mesh floor, Von Frey filament was applied to the planter aspect of right hind paw. Enough force of filament was applied against paw (causing slight bending) and hold for sec. Application of varying force (gm) repeated 6 times at interval of $4-5 \mathrm{sec}$. Withdrawal of paw was considered as a positive response. ${ }^{14}$

b) Cold Allodynia (Cold plate test): Here, the rodent was placed on the cooled plate at desired temperature $\left(5^{\circ} \mathrm{C}\right)$ and the time to induce nociceptive behaviour indicated by shivering and paw licking was recorded as the response time. ${ }^{15}$

c) Mechanical Hyperalgesia (Randall Sellitto method): Randall Selitto test is commonly used for testing acute mechanical sensitivity, measured by paw withdrawal threshold. Through the dome-shaped plastic tip of this apparatus, steadily increasing pressure applied on dorsal surface of the rat's hind paw. The withdrawal threshold (in \% CBK) for each paw was recorded. Measurements repeated 2 or 3 times on each paw. ${ }^{16}$ Animal was held to immobilize it. Place gently the paw on the apparatus and allow the tip of device to apply on paw with application of increasing mechanical force 
and withdrawal latency to the pressure supported was noted down. ${ }^{17}$

d) Heat hyperalgesia (Hot plate test): Eddy's hot-plate was used to study the thermal nociceptive threshold by keeping the temperature at $55 \pm 2{ }^{\circ} \mathrm{C}$. Animal individually tested by placing on the hot plate and paw licking latency (sec.) was recorded. Test cut-off time of $20 \mathrm{sec}$ was maintained. ${ }^{18}$

Evaluation of Motor Nerve Conduction Velocity (MNCV): MNCV recording were carried out at the end of treatment. Animals were anesthetized by Ketamine $(90 \mathrm{mg} / \mathrm{kg}$ i.p) and Xylazine (5mg/kg i.p). MNCV assessment was done by using 8 channel powerlab (AD Instruments) with animal nerve stimulating electrode (MLA0320) and needle electrodes (MLA1204). Action Potential was generated by applying stimulating electrode at proximal end and recording done from distal end. (PowerLab setup as frequency: $10 \mathrm{~Hz}$, duration: $0.1 \mathrm{~ms}$, amplitude: $4 \mathrm{~V}$ ). The distance between the stimulating electrode and recording electrodes divided by latent period is calculated as conduction velocity. Latent period considered as the time between applications of stimulus until the peak of the action potential. ${ }^{19}$

On Next day, before scarification, animals were anaesthetized lightly with ketamine for retro-orbital collection of blood in different tubes. Serum and plasma separated for electrolyte and cytokine estimation.

\section{Antioxidant study}

a) Preparation of tissue homogenate: After scarification sciatic nerve isolated and homogenized in ice cold TrisHCl buffer $(10 \mathrm{mM}, 10 \% \mathrm{w} / \mathrm{v})$. Centrifugation (using Remi C-24 high speed cooling centrifuge) carried out at 10,000 rpm for $15 \mathrm{~min}$. Clear supernatant was used for further estimations. ${ }^{11}$

b) RGSH (Reduced Glutathione): It is determined by DTNB reagent method. The colour intensity developed was measured at $412 \mathrm{~nm}$ against reagent blank. ${ }^{20}$

\section{Oxidative stress determination: MDA}

(Malondialdehyde): It is determined by TBARS method. Absorbance of organic phase recorded at $535 \mathrm{~nm} .{ }^{21}$

Histopathology: Isolatedsciatic nerve was kept in the $10 \%$ formalin and sent for study in pathology lab. ${ }^{22}$

C-reactive protein (CRP): It is determined by immunoenzymatic method.

Insulin Assay: Serum insulin $(\mu \mathrm{IU} / \mathrm{mL})$ is determined by CLIA method.
Serum electrolytes: Serum sodium $\left(\mathrm{Na}^{+}\right)$and potassium $\left(\mathrm{K}^{+}\right)$levels $(\mathrm{mmol} / \mathrm{L})$ are determined by direct ion selective electrode method.

TNF- $\alpha$, IL-6 and INF-- $\boldsymbol{\gamma}$ : It is estimated by MACSPlex cytokine 12 kit, developed for the simultaneous flow Cytometric detection of cytokines.

\section{RESULTS AND DISCUSSION}

DN is a common as well as most concerned complication, occurring in almost $50 \%$ of diabetes patients. Round about 10 to $20 \%$ of diabetic population may experience painful symptoms and 40 to $50 \%$ of them with DN experience chronic pain of neuropathy. ${ }^{23}$ Within 72 hrs of STZ injection, animals show hyperglycemia and lowered insulin secretion. STZ treatment exhibit behavioural signs of $\mathrm{DN}$ i.e. reduction in mechanical pressure withdrawal threshold and thermal withdrawal latency after about 2 weeks of injection. ${ }^{23}$

Blood glucose level: After $72 \mathrm{hr}$. of STZ injection, blood glucose level was found to be increased significantly in all treatment groups. Treatment with syringic acid 12.5, 25, $50 \mathrm{mg} / \mathrm{kg}$ and of Sinapic acid 5, $10,20 \mathrm{mg} / \mathrm{kg}$ orally for 5 weeks has shown to reduce blood glucose level. SY1, SP1, std drug reduced it but statistically non-significant. SY2 and SP2 effect is with $p<0.05^{*}$ and i.e. of SY3 and SP3 with $p<0.01^{* *}$. Thus, syringic acid and sinapic acid have shown antihyperglycemic effect (Figure 1).

Mechanical allodynia (Von Frey test): Allodynia term refers to pain due to normally non noxious stimuli. It approximately occurs in 30-50\% of diabetic patients. This can be studies in STZ induced animal model, where nociceptive behaviour can be provoked by minimum force of Von Frey filaments to the paw (maximum upto15 g). Nitrosative stress, poly (ADP-ribose) polymerase (PARP) activation, increased excitability of ganglion neurons were thought to be associated with allodynia. $^{23}$

Observations of mechanical allodynia are noted with 6 repeated application of varying force of Von Frey filament. Observations are recorded in the format of OXXOXO, where $\mathrm{O}$ indicated- no withdrawal response and $\mathrm{X}$ indicates withdrawal response. ${ }^{24} \mathrm{It}$ is observed in positive control group that hyperalgesia is produced from $1^{\text {st }}$ week indicated by withdrawal response to minimum force of filament. Treatment with SY and SP has shown to increase the threshold and force giving withdrawal response is found to be increased in dose dependent manner with change in observation format. 
Cold Allodynia (Cold plate test): Paw withdrawal latency from cold plate $\left(5^{\circ} \mathrm{C}\right)$ is found to be decreased in positive control group than negative control group. Symptoms of cold allodynia were observed from $3^{\text {rd }}$ week of diabetes. SY1 $\left(p<0.05^{*}\right)$, SY2, SY3 and SP1, SP2, SP3 and Std drug gabapentin decreased cold allodynia with $p<0.01^{* *}$ (Figure 2). These test drugs have shown protective effect from $1^{\text {st }}$ week of treatment.

Mechanical hyperalgesia (Randall Selitto test): STZ induced diabetic animal model is widely accepted for study of mechanical hypersensitivity. An increased withdrawal threshold after mechanical pressure was considered to be associated with aldose reductase (AR) and oxidative stress. ${ }^{23}$

Withdrawal threshold of paw pressure is measured in terms of \% CBK by Randall-Selitto apparatus. \% CBK is found to be decreased in positive control group from $3^{\text {rd }}$ week of diabetes induction. 5 week treatment with SY1, SY2 and SP1, SP2 has shown protective effect

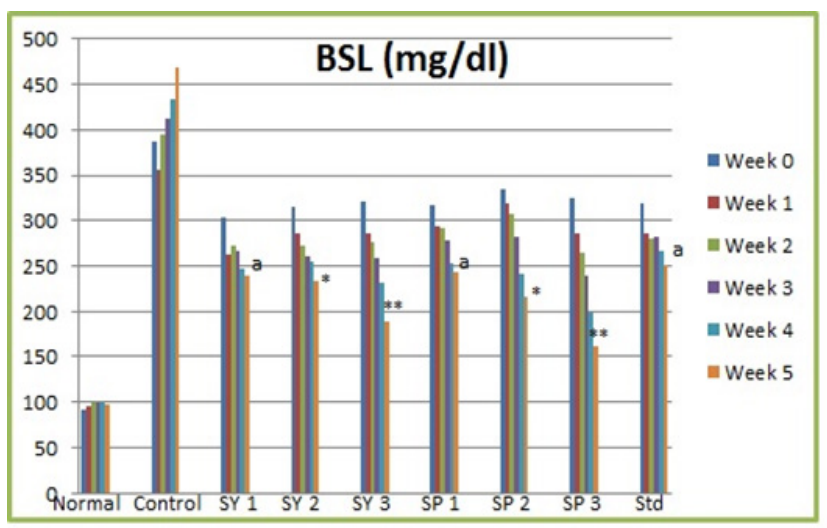

Figure 1: Result of weekly Blood Glucose Level $(\mathrm{mg} / \mathrm{dl})$ in control and treated groups. $\left({ }^{*} p<0.05,{ }^{* *} p<0.001\right.$ and $a$ is statistically non-significant compared to positive control group)

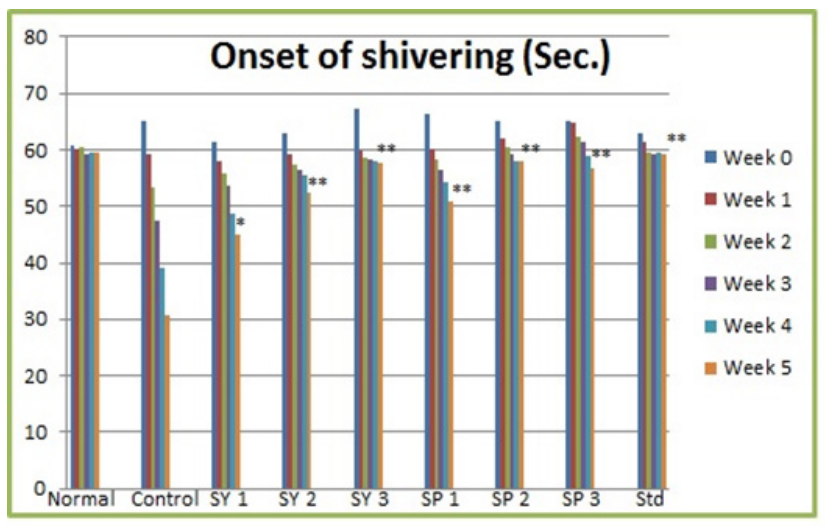

Figure 2: Result of weekly Cold Plate Test (Time in seconds indicating onset of shivering chills and paw licking). $\left(p<0.05^{*}\right)$ indicted by dose dependent increase in $\% \mathrm{CBK}$ and SY3, SP3 with $p<0.01 * *$ (Figure 3). All observations are compared with standard gabapentin $\left(p<0.01^{* *}\right)$.

Heat hyperalgesia (Hot plate test): It is an indicative of thermal hypersensitivity, which probably due to nociceptors or peripheral nerves damage. In STZ induced DN, mechanisms of thermal hyperalgesia are considered as increased oxidative stress and increased activity of protein kinase $\mathrm{C}$ (PKC), aldose reductase (AR), poly (ADP-ribose) polymerase (PARP), angiotensin converting enzyme (ACE) and toll-like receptor $4 .^{23}$

Paw withdrawal latency and jumping response was observed after placing animal on preheated plate $\left(55^{\circ} \mathrm{C}\right)$. Thermal hyperalgesia is found to be produced in positive control group from $2^{\text {nd }}$ week of diabetes. Treatment with SY and SP has prevented thermal hyperalgesia from $1^{\text {st }}$ week of treatment. After 5 weeks treatment SY2, SY3, SP1, SP2, SP3 and std drug shown significant protection $(p<0.01 * *)$ of thermal

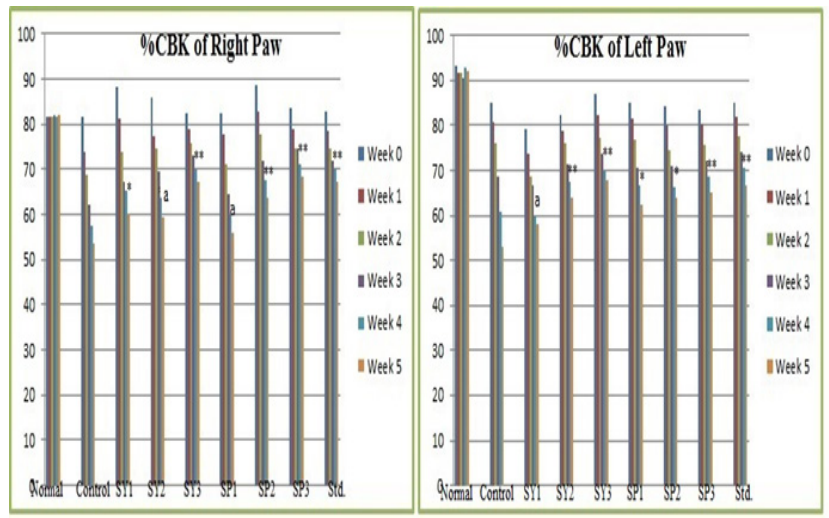

Figure 3: Result of weekly Randell Sellito Test (Right and left paw withdrawal pressure in \%CBK).

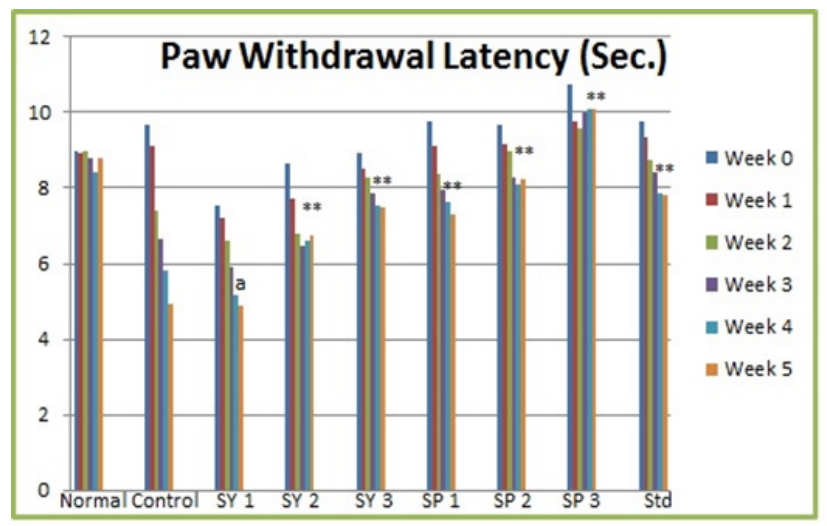

Figure 4: Result of weekly Hot Plate Test (Time in seconds indicating paw licking on hot plate of temperature $55^{\circ} \mathrm{C}$ ). 
hyperalgesia. SY1 has shown protection but statistically non-significant (Figure 4).

Motor Nerve Conduction Velocity (MNCV): After completion of $5^{\text {th }}$ week's treatment, MNCV $(\mathrm{m} / \mathrm{sec})$ was measured. Positive control animals have shown marked reduction in conduction velocity compared to negative control animals. This indicates neuronal damage which is protected in treatment groups. Treatment with SY1, SP2 $\left(p<0.05^{*}\right)$ SY2, SY3,SP3, stdhas prevented this damage and shown to increase conduction velocity (with $p<0.01^{* *}$ ) than positive control group. SP1 dose have increased MNCV but statistically non-significant (Figure 5).

Abnormalities in motor nerve conduction are associated with reduction in neuronal blood flow induced by hyperglycemia and such resultant endoneural hypoxia lead to development of diabetic neuropathy. ${ }^{25}$

Oxidative stress (MDA): Malondialdehyde (MDA) was measured from sciatic nerve tissue homogenate and absorbance recorded at $535 \mathrm{~nm}$. Oxidative stress is found to be increased in positive control group indicated by increased absorbance. The MDA absorbance of positive control was considered as $100 \%$ (i.e. $0 \%$ inhibition of MDA) and comparatively $\%$ inhibition calculated for test and standard groups. Absorbance is comparatively decreased and $\%$ inhibition is increased significantly by SP1 $\left(p<0.05^{*}\right)$ and in SY3, SP2, SP3 and standard group with $p<0.01^{* *}$. (Figure 6). SY1 and SY2 have shown statistically non-significant effect. This indicated that SY and SP are able to reduce oxidative stress, in dose dependant manner, generated by STZ in neuropathy.

Antioxidant (GSH): It is abundantly available cellular antioxidant. In diabetes hyperglycemia induces glutathione (GSH) depletion and impaired regeneration which links to diabetic neuropathy like complications. ${ }^{26}$

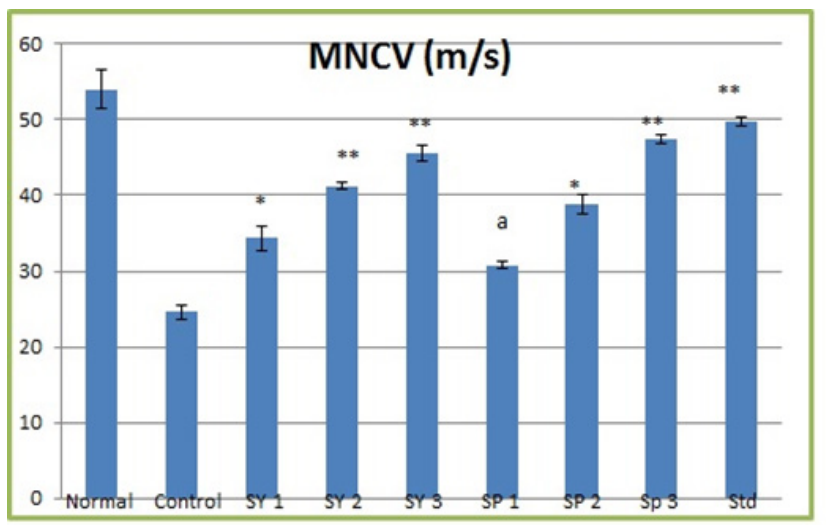

Figure 5: Nerve Conduction Velocity $(\mathrm{m} / \mathrm{s})$ after 5 week treatment in diabetic neuropathy $\left({ }^{*} p<0.05,{ }^{* *} p<0.001\right.$ and a is statistically non-significant compared to positive control group).
After STZ injection and neuropathy development GSH level is found to be decreased in positive control group indicted by significant decrease in absorbance at 412 $\mathrm{nm}$. This absorbance is increased in treatment group's dose dependently. \% increase in GSH level is calculated by considering $100 \%$ GSH level of negative control and $0 \%$ in positive control group. This study indicates 5 week treatment with SY and SP (SY2, SP2 with $p<0.05)$ is able to increase antioxidant GSH level in cell (Figure 7). A dose of SY1 and SP1 increased GSH level statistically non-significant. SY3, SP3 and std drug improved GSH with $p<0.01^{* *}$.

Thus, STZ induced DN is widely studied animal model. Possibly responsible mechanisms for hyperalgesia and abnormal sensation are increased oxidative-nitrosative stress and AR, PKC, PARP and ACE activations. It may also be associated with C-peptide deficiency, impaired neurotrophism and proinflammatory response. ${ }^{23}$

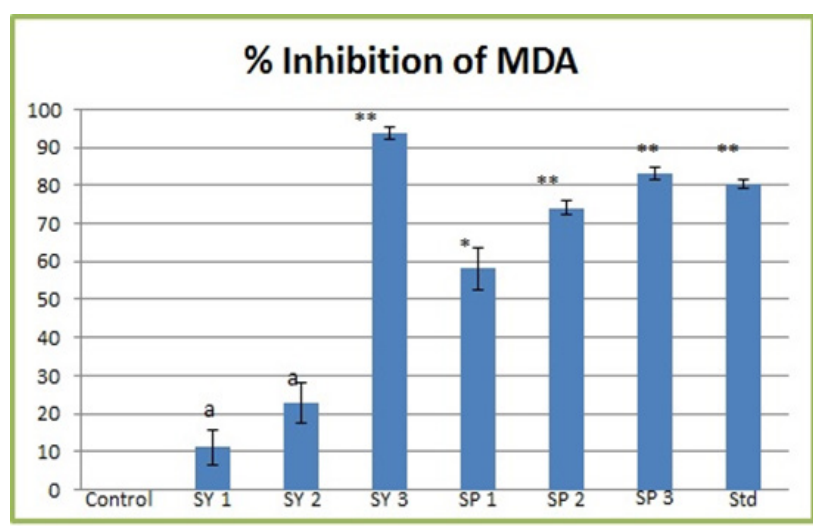

Figure 6: Percent inhibition of lipid peroxidation in terms of malondealdehyde (MDA) ( ${ }^{*} p<0.05,{ }^{* *} p<0.001$ and $a$ is statistically non-significant compared to positive control group).

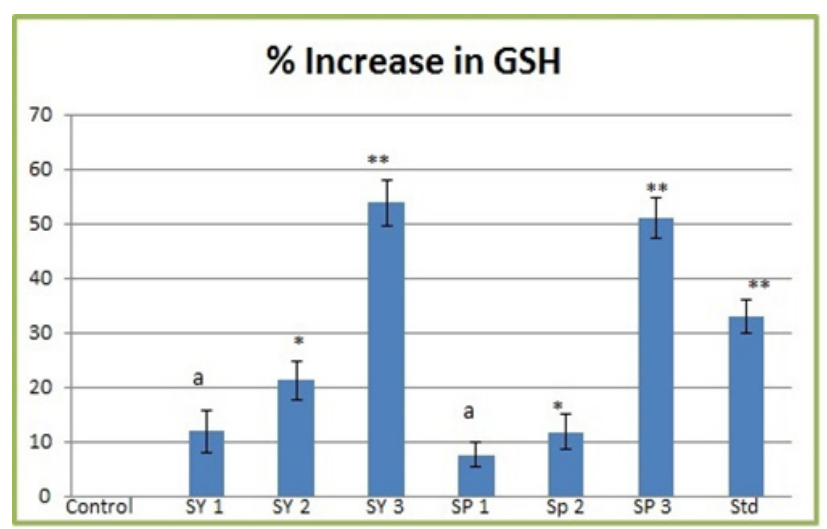

Figure 7: Percent increase in antioxidant in terms of glutathione (GSH). 
C-reactive proteins (CRP): CRP is an important pro-inflammatory factor, regulated by interleukin-6, interleukin -1 and TNF- $\alpha$. During inflammation, it is produced by the liver. ${ }^{27}$ It is determined by immunoenzymatic method. It is found that serum CRP is comparatively enhanced in positive control group than negative control, indicating possibility of neuronal damage and neuroinflammation. 5weeks treatment with SY2, SY3, SP2, SP3 and Std gabapentin reduced CRP with $p<0.01^{* *}$. SY1 and SP1 have shown statistically non-significant protective effect indicated by decrease in CRP compared to positive control group (Figure 8).

Insulin Assay: Serum insulin $(\mu \mathrm{IU} / \mathrm{mL})$ is determined by CLIA method. In positive control animals insulin level is found to be decreased significantly indicating damage to $\beta$ pancreatic cells. This is also indicated by severe hyperglycaemia. Blood sugar level is found to be controlled by treatment with SY and SP supported by rise in serum insulin level $\left(p<0.05^{*}\right)$. SP1 gives non-significant increase and SY3 shown increase with

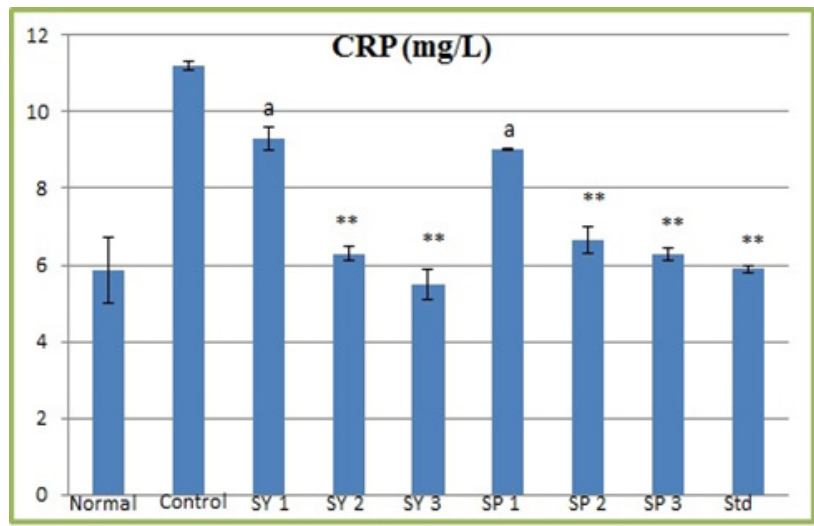

Figure 8: Serum C- reactive protein (CRP) level in treated groups compared to positive control group at the end of treatment schedule.

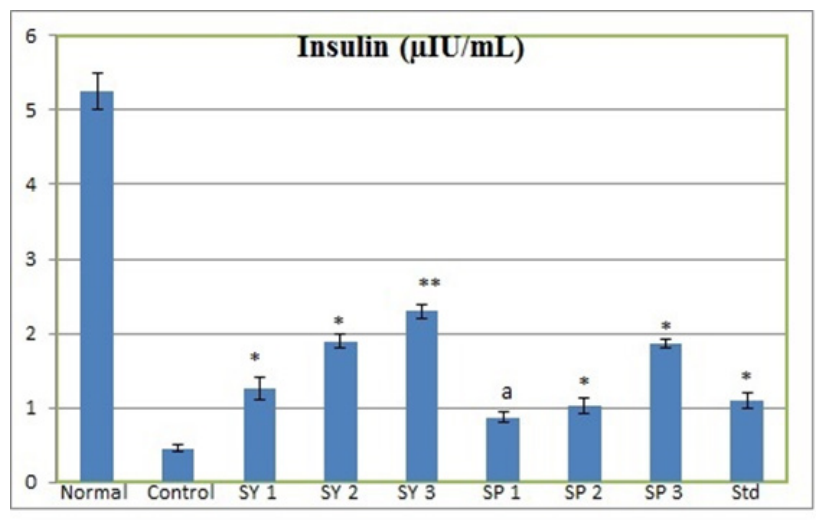

Figure 9: Serum insulin level after 5 week treatment in diabetic animals. $p<0.01^{* *}$. Thus 5 weeks treatment with SY and SP has shown protective effect on $\beta$ pancreatic cells (Figure 9).

Tumour Necrosis Factor- $\alpha$, Interleukin-6 and Interferon- $\boldsymbol{\gamma}$ : STZ-injected diabetic rats had significantly increased IL-6, TNF- $\alpha$ and INF- $\gamma$ as compared to negative control rats. Gabapentin, SY and SP treatment have shown significant $(p<0.01 * *)$ decrease in IL-6, TNF- $\alpha$ and INF- $\gamma$ (Figure 10). Inflammatory pathology in diabetes can be confirmed by increase in infiltration of inflammatory cells and changes in cytokines levels. ${ }^{28}$ Oxidative stress causes production of abnormal cytokine production (TNF$\alpha$, IL-6 and INF- $\gamma) \cdot{ }^{27}$ Previous studies reported direct inter-relation of TNF- $\alpha$, IL-1and IL- 6 with insulin. Also, it is suggested that inflammation can directly cause insulin resistance. TNF- $\alpha$ decreases insulin secretion by causing inflammation. ${ }^{27}$

Serum electrolytes: Serum electrolyte level is changed with plasma glucose level. Electrolyte balance is disturbed with diabetes melliatus. Consistent hyperglycemia can damage $\mathrm{Na}^{+}-\mathrm{K}^{+}$ATPase and other ATPase pumps and causes reduction in serum $\mathrm{Na}^{+}$level. ${ }^{29}$ Serum sodium $\left(\mathrm{Na}^{+}\right)$and potassium $\left(\mathrm{K}^{+}\right)$levels $(\mathrm{mmol} / \mathrm{L})$ are determined by direct ion selective electrode method at Apollo diagnostic lab. Comparatively serum $\mathrm{Na}^{+}$level is found to be decreased and $\mathrm{K}^{+}$level is increased in positive control animals than negative control. 5 weeks treatment with SY and SP have reversed the effect by increasing serum $\mathrm{Na}^{+}\left(p<0.01^{* *}\right)$ and decreasing $\mathrm{K}^{+}(\mathrm{SY} 1$ statistically non-significant, SP1, SP2 with $p<0.05^{*}$ and SY2, SY3, SP3 and std with $p<0.01 * *$ ) level indicating its neuronal protective effect (Figure 11).

Histopathology: Histopathology Section of $\mathrm{H}$ and E stained sciatic nerve of diabetic control rats showed epineuronaloedema and infiltration of neutrophils around blood vessels and swelling of nerve fibres

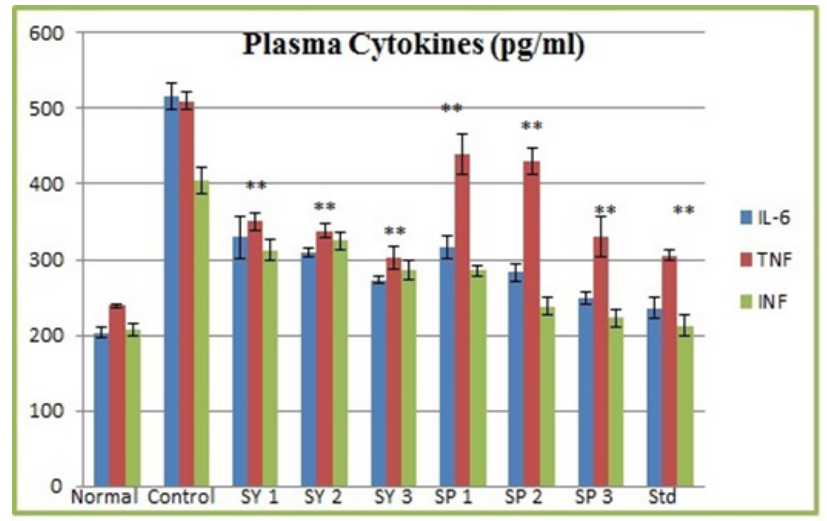

Figure 10: Plasma IL-6, TNF-a and INF-y level at the end of treatment schedule compared to positive control group ( ${ }^{\star \star} p<0.001$ compared to positive control group). 


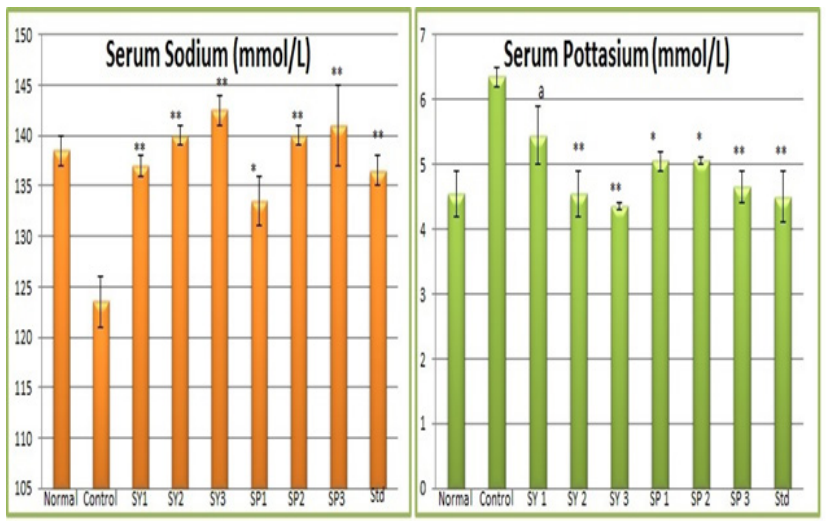

Figure 11: Serum electrolytes (Sodium and potassium) after 5 weeks treatment in diabetic animals.

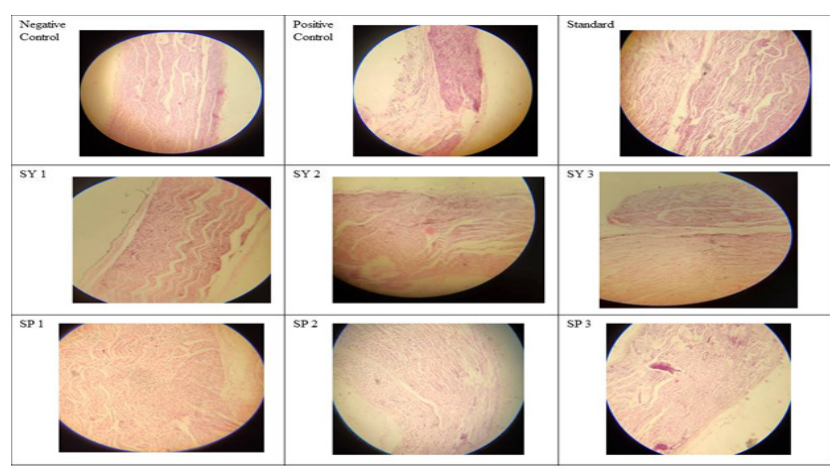

Figure 12: Histopathology Section of $\mathrm{H}$ and $\mathrm{E}$ stained sciatic nerve of diabetic control rats and treated rats.

(100x) compared to negative control group (Figure 12). Treatment with SY, SP and Gabapentin showed mild oedema and few infiltrating neutrophils around blood vessels and minor swelling of nerve fibres.

Biostatistics: Results are expressed as mean \pm S.D. Statistical analysis was performed using Graph Pad Prism Software version 8. For multiple comparisons, one-way Analysis of Variance (ANOVA) was used followed by with Dunnet test. $p<0.05^{*}$ and $p<0.01 * *$ are considered statistically significant and a is statistically non-significant as compared to positive control group.

\section{CONCLUSION}

From above results, it can be concluded that Syringic acid and Sinapic acid have neuroprotective role in diabetic neuropathy. This effect may be attributed to their anti-hyperglycemic, anti-hyperalgesic, antioxidant and anti-inflammatory action.

\section{ACKNOWLEDGEMENT}

Authors are thankful to trust of MGV and SNJB for providing necessary laboratory facilities.

\section{CONFLICT OF INTEREST}

The authors do not have any conflict of interest.

\section{ABBREVIATIONS}

DN: Diabetic Neuropathy; STZ: Streptozotocin; DM: Diabetic Mellitus; SY: Syringic acid; SP: Sinapic acid; MNCV: Motor Nerve Conduction Velocity; MDA: Malondialdehyde; GSH: Glutathione; CRP: C-Reactive protein; TNF: Tumour Necrosis Factor; INF: Interferon.

\section{REFERENCES}

1. Angela MS, Carla LP, Alexandre S. Experimental models for the study of neuropathic pain. Rev Dor Sao Paulo. 2016;17(1):27-30.

2. Forouzanfar F, Hosseinzadeh $\mathrm{H}$. Medicinal herbs in the treatment of neuropathic pain: A review. Iran J Basic Med Sci. 2018;21(4):347-58.

3. Deeds MC, Anderson JM, Armstrong AS, Gastineau DA, Hiddinga HJ, Jahangir A, et al. Single Dose Streptozotocin Induced Diabetes: Considerations for Study Design in Islet Transplantation Models. Lab Anim. 2011;45(3):131-40.

4. Ozyurt B, Gulec M, Ozyurt H, Ekici F, Atis O, Akbas A. The effect of antioxidant caffeic acid phenethyl ester (CAPE) on some enzyme activities in cisplatininduced neurotoxicity in rats. Eur J Gen Med. 2006;3(4):167-72.

5. Szwajgier D, Borowiec K, Pustelniak K. The Neuroprotective Effects of Phenolic Acids: Molecular Mechanism of Action. Nutri. 2017;9(5):477-98.

6. Cheemanapalli S, Mopuri R, Golla R, Anuradha C, Chitta S. Syringic acid (SA): A Review of its Occurrence, Biosynthesis, Pharmacological and Industrial Importance. Biomed and Pharmacother. 2018;108:547-57.

7. Raish M, Ahmed A, Ansari MA, Alkharfy KM, Ahada KA, et al. Effect of sinapic acid on aripiprazole pharmacokinetics in rats: Possible food drug interaction. $\mathrm{J}$ of Food and Drug Anal. 2019;27(1):332-8.

8. Shahmohamady P, Eidi A, Mortazavi P, Panahi N, Minai-tehrani D. Effect of sinapic acid on memory deficits and neuronal degeneration induced by intra cerebroventricular administration of streptozotocin in rats. Pol J Pathol. 2018;69(3):266-77.

9. Khadke SS, Pachauri DR, Mahajan SD. An Acute Oral Toxicity Study of Gnidia glauca (Fresen.) Gilg in Albino Rats as per OECD Guideline 425. Interna J Pharm Tech Res. 2011;3(2):787-91.

10. Ataroalsadat M, Abdodlah A, Seyed KG, Ramin P, Mohammad B. The effects of dosage and the routes of administrations of streptozotocin and alloxan on induction rate of type 1 diabetes mellitus and mortality rate in rats. Lab Anim Res. 2016;32(3):160-5.

11. Nithya M, Ambikapathy V, Panneerselvam A. In vivo Antioxidant and Enzymatic Activity of Ganoderma lucidum (Curt:fr.) P. Karst. on Mammary Cells of DMBA Induced Sprague dawley Rats. Int J Curr Microbiol App Sci. 2015;4(6):69-77.

12. Alireza N, Mohammad B, Mohsen S, Ali F, Azim A. Attenuation of oxidative stress in streptozotocin-induced diabetic rats by Eucalyptus globulus. Ind J Clin Biochem. 2009;24(4):419-25.

13. Wang LX, Wang ZJ. Animal and cellular models of chronic pain. Adv drug Deliv Rev. 2003;55(8):949-65.

14. Sanklecha D, Upaganlawar A, Upasani C. Neuroprotective Effects of Protocatechuic Acid in Diabetes Induced Neuropathic Pain. Am J Biochem Mol Biol. 2017;7(3):111-7.

15. Furgala A, Salat R, Salat K. Acute cold allodynia induced by oxaliplatin is attenuated by amitriptyline. Acta Neurobiol Exp. 2018;78:315-21.

16. Honore P. Behavioral Assessment of Neuropathic Pain in Preclinical Models. Drug Dev Res. 2006;67(4):302-7.

17. Khan D, Mubashir M, Upaganlwar A, Sangshetti JN, Upasani CD, Une HD. Quantitative assessment of tactile allodynia and protective effects of flavonoids of Ficuscarica Lam.leaves in diabetic neuropathy. Pharmacog Mag. 2019;15(62):128-34. 
18. Krishnamurthy B, Prashanth A, Sathyanarayanan A. Comparison of the Effects of Zonisamide and Flupirtine on Paclitaxel Induced Peripheral Neuropathy in Rats. J Clin Diagno Res. 2018;12(1):FC05-8.

19. EISayed LA, Eltablawy SN. Nerve Conduction Velocity of Sciatic Nerve in High Fat Diet Induced Obesity in Rats: Effect of Corn Oil and Omega 3 Fatty Acids Supplement. Life Sci J. 2012;9(3):2251-64.

20. Sajeeth $\mathrm{Cl}$, Manna PK, Manavalan R. Antioxidant Activity of Polyherbal Formulation on Streptozotocin Induced Diabetes in Experimental Animals. Der Pharmacia Sinica. 2011;2(2):220-6.

21. Bishnoi M, Patil CS, Kumar A, Kulkarni SK. Relative role of cyclooxygenase 2 (COX 2) inhibitors and lipoxygenase (LOX) inhibitors in ageing induced dementia and oxidative damage. Ann of Neurosci. 2005;12(2):6-12.

22. Memari E, Hussainian M, Mirkheshti A, Dolatabadi A, Mirabotalebi M, Khandaghy $\mathrm{M}$, et al. Comparison of histopathological effects of perineural administration of bupivacaine and bupivacaine-dexmedetomidine in rat sciatic nerve. Exp Toxicol Pathol. 2016;68(10):559-64.

23. Gao F, Zheng ZM. Animal Models of Diabetic Neuropathic Pain. Exp Clin Endocrinol Diabetes. 2014;122(02):100-6.

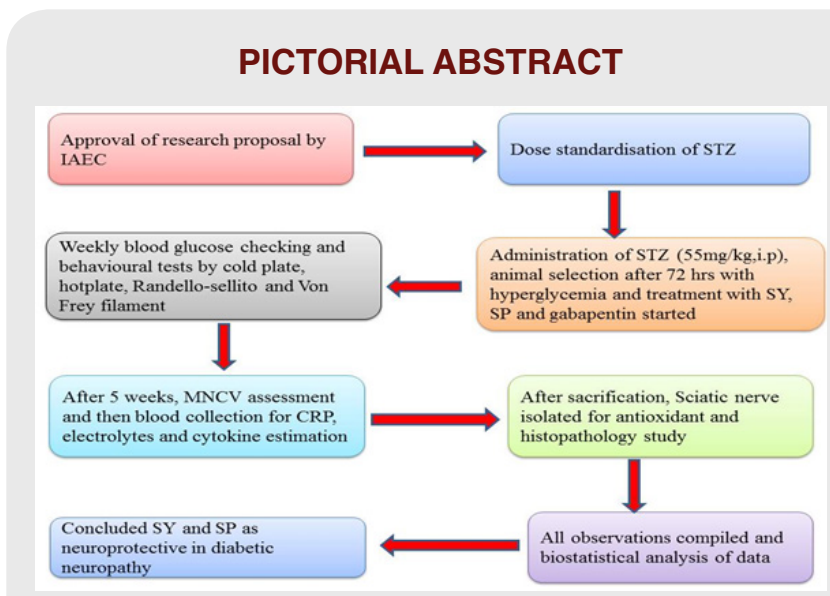

24. Chaplan SR, Bach FW, Pogrel JW, Chung JM, Yaksh TL. Quantitative assessment of tactile Allodynia in the rat paw. J Neurosci Meth. 1994;53(1):5563.

25. Coppey LJ, Davidson EP, Dunlap JA, Lund DD, Yorek MA. Slowing of motor nerve conduction velocity in streptozotocin-induced diabetic rats is preceded by impaired vasodilation in arterioles that overlie the sciatic nerve. Int J Exp Diabetes Res. 2000;1(2):131-43.

26. Yorek MA. The role of oxidative stress in diabetic vascular and neural disease. Free Radic Res. 2003;37(5):471-80.

27. Behzad M, Seyed AR, Parnia T, Seyed AS, Mehrnoush R, Fatemeh R, et al. Beneficial effects of Spirogyra neglecta Extract on antioxidant and anti-inflammatory factors in streptozotocin-induced diabetic rats. Biomol Concepts. 2018;9(1):184-9.

28. Fischer YW, Garyantes T. Improving the Reliability and Utility of StreptozotocinInduced Rat Diabetic Model. J Diab Res. 2018;1-14.

29. Wang S, Hou X, Liu Y, Lu H, Wei L, Bao Y, et al. Serum Electrolyte Levels in Relation to Macrovascular Complications in Chinese Patients with Diabetes Melliatus. Cardiovascular Diabetology. 2013;12(1):146.
In the present study, STZ have induced neuropathy symptoms in $3^{\text {rd }}$ week in positive control group. 5 weeks treatment with SY and SP has found to protect behavioural changes by reducing mechanical allodynia, thermal hyperalgesia and mechanical hyperalgesia. Oxidative stress and antioxidants enzyme level in also found to be protected in treated groups in dose dependant manner. Nerve conduction velocity was decreased in positive control group due to neuronal hypoxia produced by hyperglycemia. This was significantly improved by treatment with SY and SP. Markers of neuroinflammation i.e. $C$ reactive protein, Interleukin-6, TNF- $\alpha$ and INF- $\gamma$ were found to be increased in positive control group after STZ. These cytokine levels were found to be decreased in treatment group of SY and SP. Thus, from this result, it is concluded that Syringic acid and Sinapic acid have neuroprotective role which may be attributed to their anti-hyperglycemic, anti-hyperalgesic, antioxidant and anti-inflammatory action. Sothese natural phenolic acids syringic acid and Sinapic acid can be therapeutically used in combination with current treatment of diabetic neuropathy.

Cite this article: Pawar SH, Upaganlawar AB, Upasani CD. Evaluation of Some Phenolic Acids in Diabetic Neuropathy. Indian J of Pharmaceutical Education and Research. 2021;55(1s):s176-s183. 